\title{
Leaflet Kinematics of Mechanical and Bioprosthetic Aortic Valve Prostheses
}

\author{
Bernhard Vennemann, *† Thomas Rösgen,* Paul Philipp Heinisch,
}

\begin{abstract}
The hemodynamic performance of artificial aortic valves (AVs) and the probability for structural valve deterioration can be linked to the valve kinematics. Comparability among different studies is limited because of variations in the experimental setups and physiologic boundary conditions. This study presents results of kinematic measurements of bioprosthetic and mechanical AVs that were tested in an identical experimental setting such that they can be directly compared with each other. The kinematics of AVs is typically presented in the form of the geometric orifice area and its temporal evolution. These parameters cannot capture asynchronous leaflet motion and out-of-plane leaflet velocity. In this work, each leaflet was tracked individually for a more detailed understanding of the leaflet kinematics, asynchronous leaflet motion, and leaflet tip velocities. A bioprosthetic valve, Edwards INTUITY (EINT), and two mechanical valves, Medtronic ADVANTAGE (MADV) and a Lapeyre-Triflo FURTIVA (TFUR), were tested in a compliant model of the aortic root in a physiologic flow loop. TFUR and MADV opened alike with maximum leaflet tip velocities of 0.77 and $0.66 \mathrm{~m} / \mathrm{s}$, respectively. The opening of EINT showed significantly higher local in-plane leaflet velocities of more than $2 \mathrm{~m} / \mathrm{s}$. EINT and TFUR exhibited similar early and slow closure. MADV closed significantly later with increased velocity. TFUR had a median maximum leaflet tip velocity of $0.39 \mathrm{~m} / \mathrm{s}$ during valve closure and that of MADV was $0.83 \mathrm{~m} / \mathrm{s}$, whereas EINT exhibited a median maximum local in-plane leaflet velocity of $0.37 \mathrm{~m} / \mathrm{s}$. EINT experienced leaflet fluttering during systole with a flapping frequency of $36 \mathrm{~Hz}$. ASAIO Journal 2017; XX:00-00.
\end{abstract}

Key Words: leaflet kinematics, heart valve, geometric orifice area, hemodynamics, rapid valve closing time, leaflet fluttering, Edwards Intuity, Lapeyre-Triflo Furtiva, Medtronic Advantage

From the *Institute of Fluid Dynamics, ETH Zürich, Sonneggstrasse 3, 8092 Zurich, Switzerland; †ARTORG Center, University of Bern, Murtenstrasse 50, 3008 Bern, Switzerland; and Department of Cardiovascular Surgery, University Hospital Bern, Bern, Switzerland.

Submitted for consideration March 2017; accepted for publication in revised form August 2017

All authors declare that they have no conflict of interest that may bias this work. The research in this work was funded with internal funds from ETH Zürich and the University of Bern.

Supplemental digital content is available for this article. Direct URL citations appear in the printed text, and links to the digital files are provided in the HTML and PDF versions of this article on the journal's Web site (www.asaiojournal.com).

Correspondence: Bernhard Vennemann, Institute of Fluid Dynamics, ETH Zürich, Sonneggstrasse 3, 8092 Zurich, Switzerland. Email: vennemann@ifd.mavt.ethz.ch

Copyright (C) 2017 by the ASAIO

DOI: 10.1097/MAT.0000000000000687
Cavitation, excessive structural load on the leaflets, and an increased closing volume together with a high-velocity regurgitant jet can be linked to unphysiological kinematics of prosthetic aortic valves (AVs). ${ }^{1-7}$ This affects their durability with premature leaflet wear or even mechanical failure and the hemodynamic performance because of increased shear stress, valvular insufficiency, regurgitation, and increased flow resistance. ${ }^{8,9}$ Comparability between different studies and different heart valves is limited because of differences in the experimental setups that affect the kinematics of the valves. In this work, a study on the leaflet kinematics of three different types of AV prostheses is presented. A bioprosthetic valve, Edwards INTUITY (EINT), a mechanical bileaflet valve, Medtronic ADVANTAGE (MADV), and a novel trileaflet mechanical valve, Lapeyre-Triflo FURTIVA (TFUR), were tested. They were inserted into an identical in vitro setup featuring a compliant model of the aortic root and were tested under the same physiologic flow conditions, allowing for a direct comparison of the results. TFUR differs from traditional mechanical AV designs because of its novel hinge mechanism and its trileaflet design, with leaflets made from polyether ether ketone, while traditional mechanical AVs, for example, MADV, feature a bileaflet design with pyrolytic carbon leaflets. ${ }^{10}$ The TFUR design aims at reduced thrombogenicity (not requiring anticoagulation therapy) while maintaining the high durability of mechanical valves. ${ }^{10,15-20}$

The geometric orifice area (GOA) and its evolution over a pulse cycle is a frequently used metric for studying the kinematics of heart valves. ${ }^{11-14}$ Although being valuable for a global analysis of the valve kinematics, this parameter has some limitations that are often overlooked: GOA is unable to capture asynchronous leaflet motion and does not provide any information on the out-of-plane velocity component of the leaflet motion. In this study, in addition to GOA, the kinematics of each individual leaflet of the AVs were measured to gain a better understanding of the detailed kinematics of AV prostheses.

To the authors' best knowledge, this is the first study where the kinematics of the TFUR valve are directly compared with the kinematics of typical mechanical and bioprosthetic AV prostheses.

\section{Materials and Methods}

\section{Experimental Setup}

The aim of the experimental setup was to provide a modular platform to test the different valves, mechanical and bioprosthetic, in an identical experimental setting. Multiple independent measurements were recorded for each $\mathrm{AV}$, and quantitative values are presented by the median values and the corresponding interquartile range. 

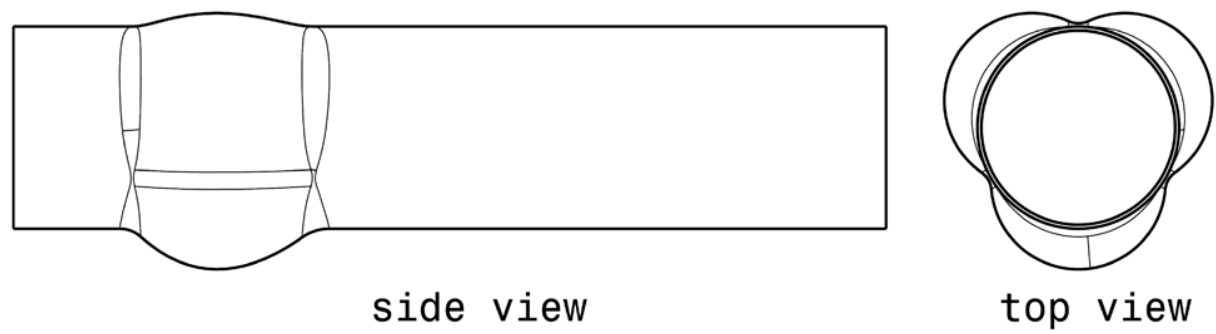

Figure 1. Geometry of the aortic root model, including the three aortic cusps.

\section{Flow Loop}

A mock loop was used to replicate physiologic flow and pressure profiles in the aortic root (a detailed description can be found in Jahren et al. ${ }^{21}$ ). The AVs were inserted into a compliant model of the aortic root in their recommended position and orientation. The compliance of the aortic root is believed to have an influence on the kinematics of the heart valve. ${ }^{22,23}$ Here, the compliance was $0.13 \% / \mathrm{mm} \mathrm{Hg}$, which corresponds to a human aortic root at 60 to 80 years of age. ${ }^{21}$ The desired compliance was achieved by repeatedly adding thin layers of liquid silicone onto a three-dimensional (3D) printed model of the aortic root until the desired stiffness was reached. The root morphology was adapted from Swanson et al. ${ }^{24}$ (Figure 1). A mixture of glycerol and water $(40 / 60) \%_{w t}$ served as blood analog matching the kinematic viscosity of 3.5 cSt. All experiments were carried out for the following standard physiologic flow and pressure conditions (as suggested in the ISO 5840) resembling a human at rest: $\mathrm{HR}=72 \mathrm{bpm}, \mathrm{CO}=5 \mathrm{~L} / \mathrm{min}, p_{\text {sys }} / p_{\text {dias }}=120 / 80 \mathrm{~mm} \mathrm{Hg}$. The flow loop was driven with a piston pump designed for hemodynamic testing (Superpump, Vivitro Systems, Victoria, BC, Canada) with its physiologic flow profile setting and a ratio of systole to diastole of $1 / 2$. Figure 2 shows the profile plotted in function of dimensionless time $\tau:=t / T_{\text {cycle }}$.

\section{Optical Setup}

The design of the test section allowed for axial optical access to the AV. The scene was recorded with a high-speed camera
(FASTCAM ultima 512, Photron USA Inc., San Diego, CA) at a frame rate of $2000 \mathrm{FPS}$ and a resolution of $512 \times 512$ pixel. The camera was equipped with a macrolens (Micro Nikkor $60 \mathrm{~mm}$ f2.8, Nikon Corporation, Tokyo, Japan) to provide sufficient magnification. A continuous LED light source (LED Daylight, Power Systems GmbH \& Co. KG, Ilsfeld-Auenstein, Germany) was used for illumination of the valve.

\section{Image Processing}

The analysis of the raw images required significant image preprocessing to obtain accurate measurements of the individual leaflet kinematics.

\section{Image Stabilization}

The heart valve could move laterally within the image frame because of the compliant nature of the valve fixation in the aortic root model. This movement had to be compensated to accurately track the valve leaflets and their kinematics. A crosscorrelation-based image stabilization algorithm was applied to detect the valve movement between subsequent frames. ${ }^{25}$ Subwindows $(60 \times 60$ pixels $)$ with distinct features (e.g., stent ring) were used to correlate subsequent images. These sub-windows had to be large enough to contain unique information that could be easily identified in each frame with the best window size and location depending of the specific features of the image. The chosen window size gave good results for all tested valves. The peak in the resulting correlation map yields the relative shift

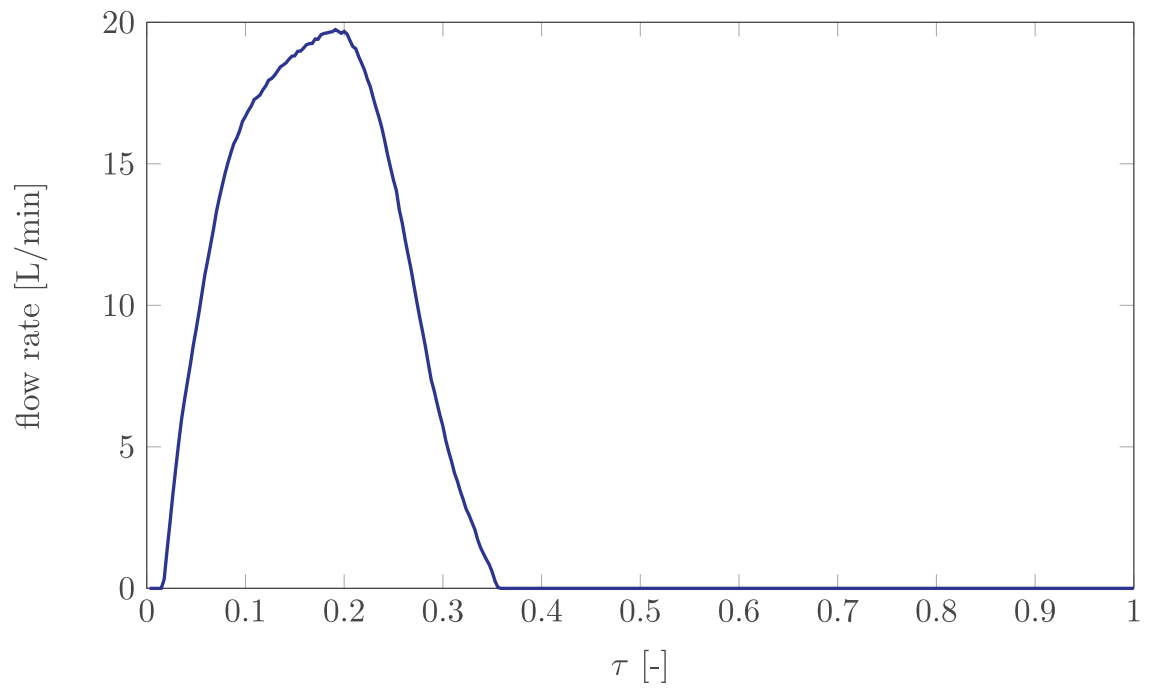

Figure 2. Physiologic pump profile of piston pump. Ratio of systole to diastole: 1/2. 


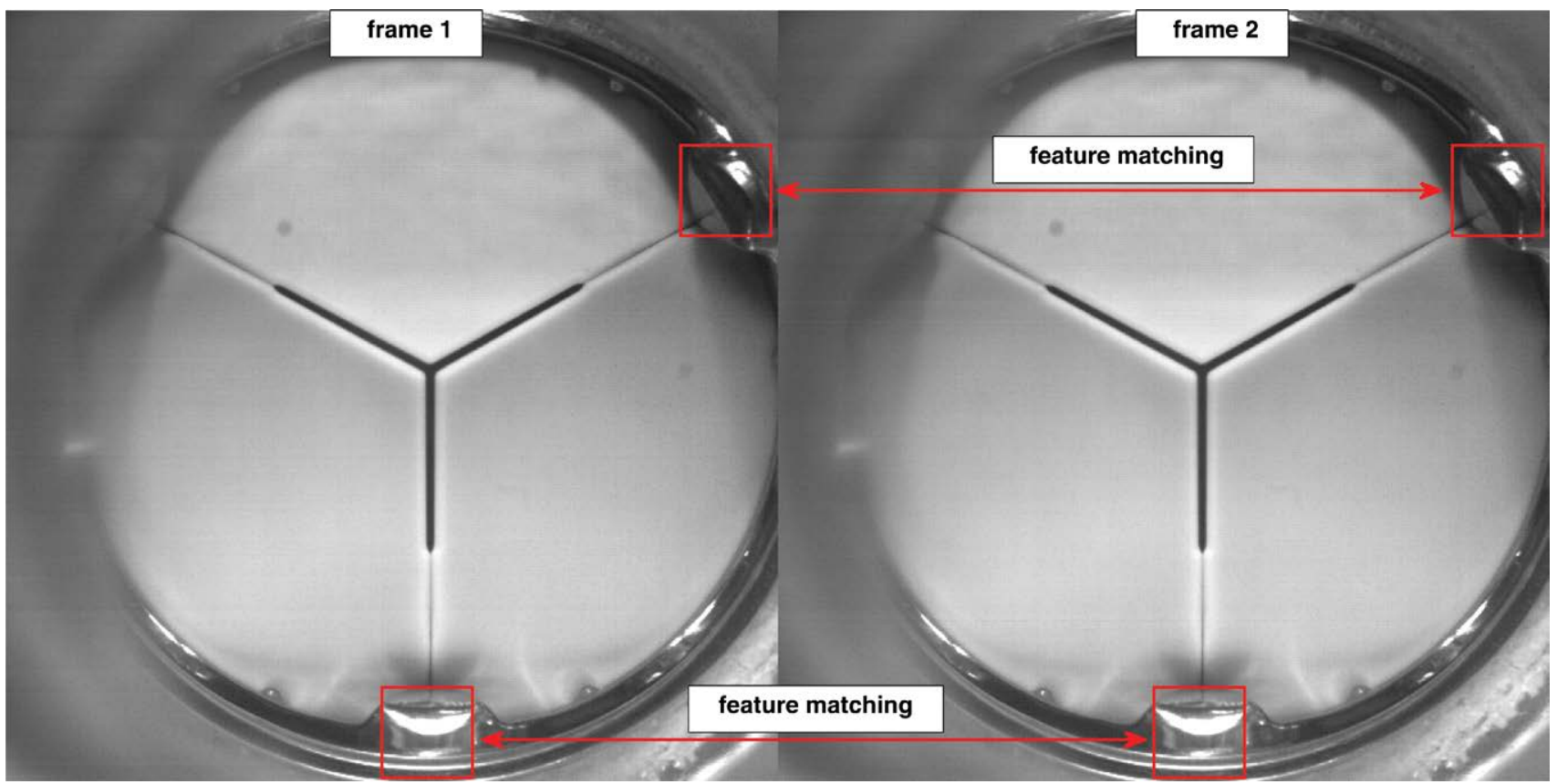

Figure 3. Feature matching for image stabilization. Identifying unique features in both frames allows to determine the relative valve movement between subsequent images and to stabilize the video sequence.

between the frames, which was used to stabilize the sequence (Figure 3). This feature-matching approach allowed to compute the relative valve movement with sub-pixel accuracy.

\section{Perspective Transformation}

The subsequent processing steps required a camera view that is well aligned with the valve axis. Careful camera positioning reduced optical skewing of the valve geometry because of camera misalignment. A perspective transformation of the image was applied to eliminate residual skewing of the raw image. This was done by identifying in each frame a set of geometrical features for which the geometric relation is known. This allowed to calculate the transformation matrix, which maps the original image to its correct perspective transformation. Figure 4 shows an example of a raw distorted image
(Figure 4A) and the perspective transformation of that image (Figure 4B). An overlay of these two images on different color channels of a composite RGB image illustrates the effect of perspective transformation (Figure 4C). The raw image in Figure 4 was captured deliberately with a poor camera alignment for better illustration. The images used for the quantitative assessment of the AV kinematics were recorded with an optimized camera alignment, leading to greatly reduced distortion.

\section{Relative Geometric Orifice Area}

Image thresholding was used to determine the GOA, which was normalized by the valve's maximum orifice area to yield the relative GOA (rGOA). This metric gives a first, global indication of the opening and closing kinematics of the valve. GOA can be used to determine average opening and closing speeds of the
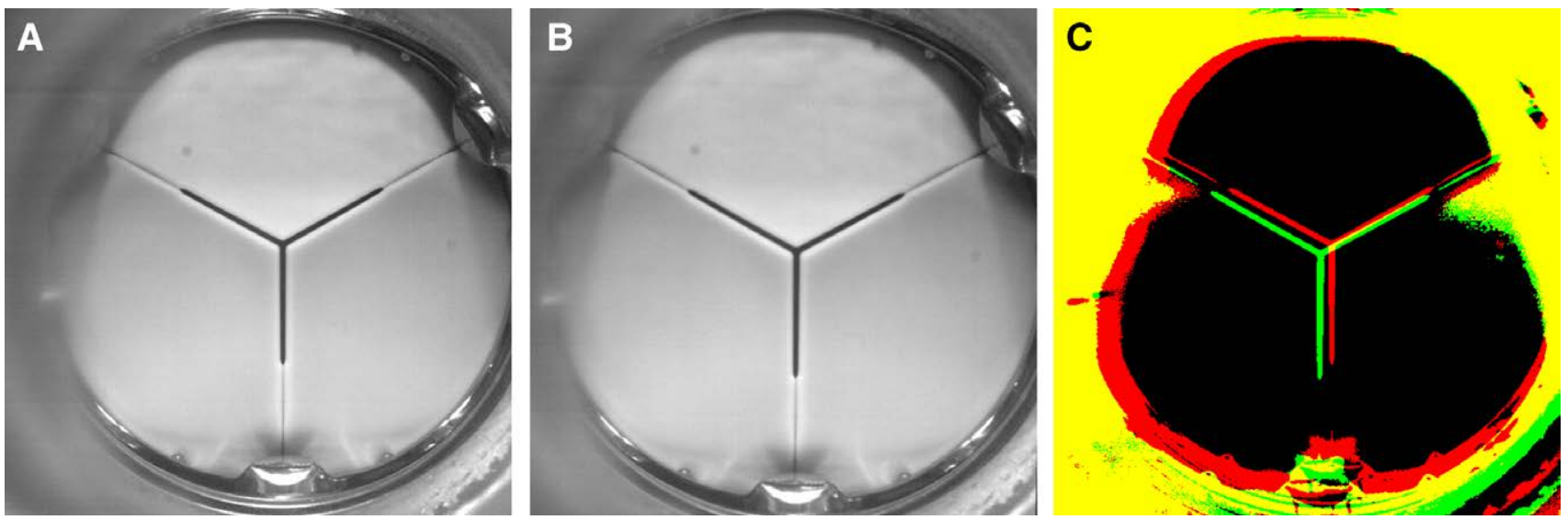

Figure 4. A perspective transformation eliminates optical distortions because of imperfect camera alignment. Original image (A), perspective transformation (B), and RGB composite image of inverted original and transformed image (C). Original image, red channel; transformed image, green channel. 
AV but cannot be used to study individual leaflet kinematics. If, for example, one leaflet opens slightly and another leaflet closes by the same amount (a situation often encountered during early valve closure), no net change in GOA can be observed.

\section{Leaflet Tracking}

By tracking the leaflets individually, asynchronous leaflet motion could be captured. The algorithm identified the contours of the leaflets based on the edge map of the image. This yielded the distances $d_{i}(t)$ of each leaflet tip to the valve center as a function of time. The distances were then translated from pixel units to physical lengths using image reference length scales (e.g., stent ring diameter).

\section{Leaflet Velocity Calculation}

For the mechanical valves, a simple mathematical model of the leaflet kinematics was developed based on the valve geometry and the location of the pivoting points. This allowed derivation of the leaflet opening angles $\varphi_{i}(t)$ from the twodimensional projections of the leaflet motion $d_{i}(t)$ as captured by the camera

$$
\phi_{i}(t)=\cos ^{-1}\left(\cos \left(\phi_{0}\right)-d_{i}(t) / L\right) \quad i=1,2,(3),
$$

where $i$ is the leaflet numbering and $L$ is the distance between the rotational axis and the leaflet tip. From these angles, all velocity components for each leaflet could be retrieved. The velocity magnitude is highest at the tip of the leaflets. It is given by

$$
V_{\mathrm{tip}, i}(t)=L \frac{\mathrm{d} \phi_{i}(t)}{\mathrm{d} t} \quad i=1,2,(3)
$$

This analysis is not possible with classical GOA calculation, which only measures the in-plane movement and, thus, underestimates the leaflet velocity.

The approach is not directly applicable to bioprosthetic valves because of their more complex kinematics and the lack of clearly defined pivoting points.

Therefore, a slightly different approach was used to obtain the kinematics of each individual leaflet of the bioprosthetic valve. The free edges of the leaflets were identified, and spline curves were fitted to create equidistant markers on each leaflet. These were then tracked over subsequent frames to yield the local in-plane velocity of the leaflets. However, this method does not capture the out-of-plane velocity component. The analysis was, thus, restricted to the in-plane velocity component, which is the dominant velocity component (because of the limited axial motion of the edges).

\section{Results}

\section{Opening and Closing Characteristics}

Figures 5 and $\mathbf{6}$ show snapshots during valve opening (Figure 5) and valve closure (Figure 6) to provide a first insight into the opening and closing characteristics of the three tested valves.

The opening phase was very similar for all three AVs starting at $t=30 \mathrm{~ms}$ and ending at $t=50 \mathrm{~ms}$. There were significant differences in the closing kinematics with respect to the onset and the duration of the closing phase. EINT and TFUR started closing at $t=270 \mathrm{~ms}$, and the valve closure was completed at $t=330 \mathrm{~ms}$ with EINT taking just a few milliseconds longer. At this point in time, MADV was still fully open. It started closing only at $t=330 \mathrm{~ms}$ and was fully closed at $t=360 \mathrm{~ms}$. Late closure of AVs may be problematic as it can lead to an increased closing volume and the generation of a high-speed regurgitant jet during valve closure. ${ }^{26}$

\section{Relative Geometric Orifice Area}

The rGOAs as function of the dimensionless time are shown in Figure 7 for a representative pulse cycle (statistical results are given in Table 1, Supplemental Digital Content, http://links. Iww.com/ASAIO/A183). The data confirms the basic observations from Figures $\mathbf{5}$ and $\mathbf{6}$.

At $\tau=0.1$, shortly after initial opening, TFUR showed a small reduction in $\mathrm{rGOA}$ of approximately $7 \%$, which then remained constant until the onset of valve closure at $\tau=0.26$. EINT showed fluttering during the fully open phase ( $\tau=0.04-0.24)$, whereas the two mechanical valves showed no such effect.

Rapid valve closure is the most critical phase during the closing process, and this period is typically referred to as rapid valve closing time (RVCT). ${ }^{8}$ RVCT was obtained by measuring the time between the onset of rapid valve movement and full valve closure and dividing by the duration of the heart cycle. Periods of rapid valve closure of the three valves are indicated by capital letters A, B, and C in Figure 7. For EINT, it started at $\tau=0.3$ and ended at $\tau=0.39$. For TFUR, it started at $\tau=0.32$ and was completed at $\tau=0.39$. Valve closure of MADV did not start until $\tau=0.39$ and it ended only at $\tau=0.425$. To further investigate the kinematic differences during the rapid valve closure, a nondimensional rapid valve closing speed (RVCS) was calculated as the ratio of the rGOA to the RVCT

$$
\text { RVCS }=\frac{\sqrt{\text { ROA }_{\text {rvct }}}}{\text { RVCT }}
$$

where $\mathrm{rGOA}_{\text {rvct }}$ is the relative GOA at the beginning of rapid valve closure (square root taken to convert area to length scale), and RVCT is the dimensionless rapid valve closing time. RVCS can be interpreted as average nondimensional closing speed of the valve. EINT had the lowest RVCS of 9.09 (0.38), followed by TFUR with 11.84 (2.40). RVCS of MADV was 24.00 (2.29), which is twice as high as that of TFUR. This corresponds directly to the rGOA of MADV exhibiting the highest gradients.

\section{Bioprosthesis Leaflet Fluttering}

A closer analysis of the fluttering of the EINT leaflets as observed in the rGOA revealed a flapping frequency of $f=36 \mathrm{~Hz}$ with a maximum amplitude of $A=2.2 \mathrm{~mm}$ (Figure 8). Here, not all leaflets were equally prone to fluttering, with one leaflet usually showing stronger fluttering than the other two. This is likely because of slight differences in the clamping of the flexible stent frame in the aortic root or small mechanical differences between the individual leaflets. 


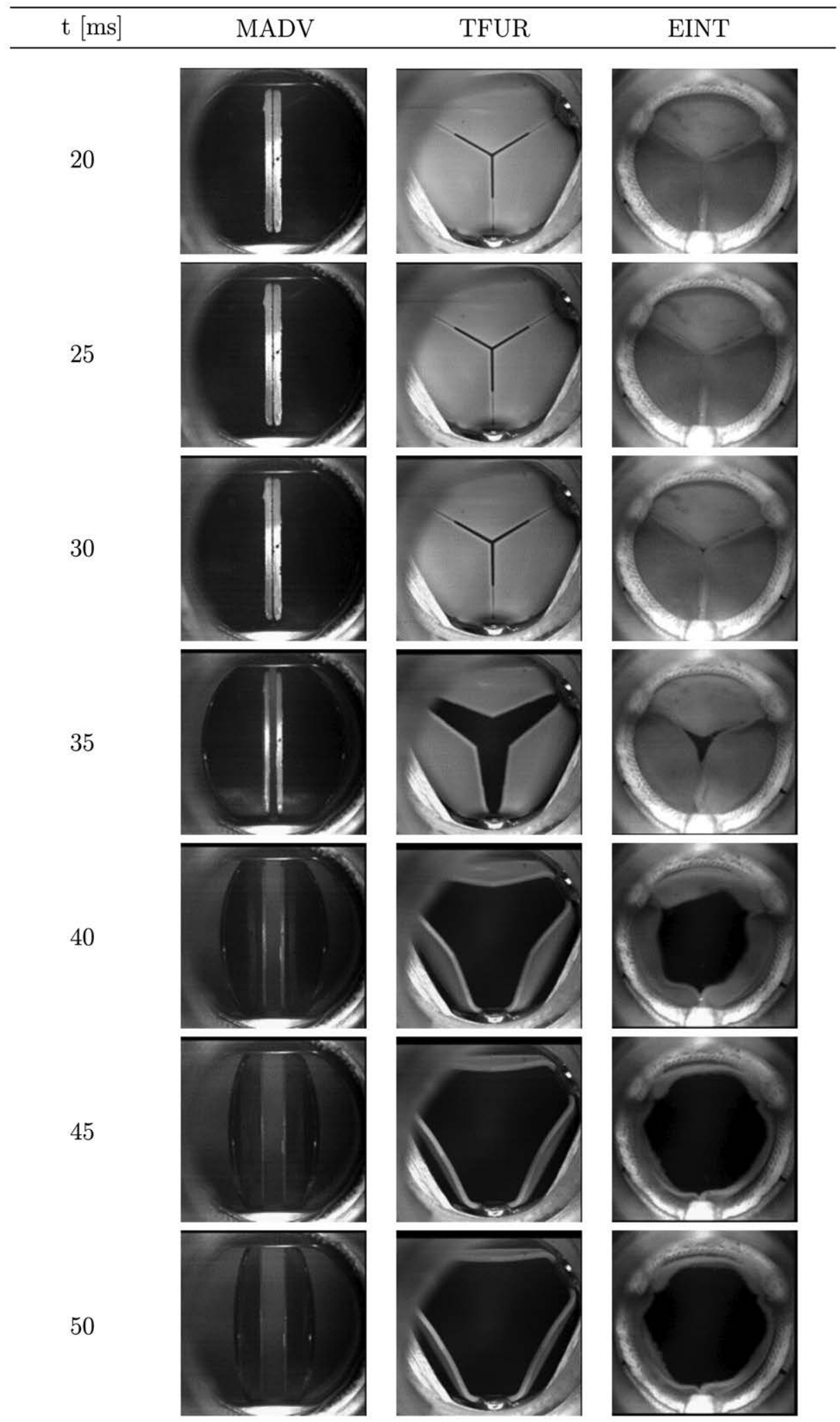

Figure 5. Qualitative comparison of the opening phase. All valves behaved alike. EINT, Edwards INTUITY; MADV, Medtronic ADVANTAGE; TFUR, Lapeyre-Triflo FURTIVA. 


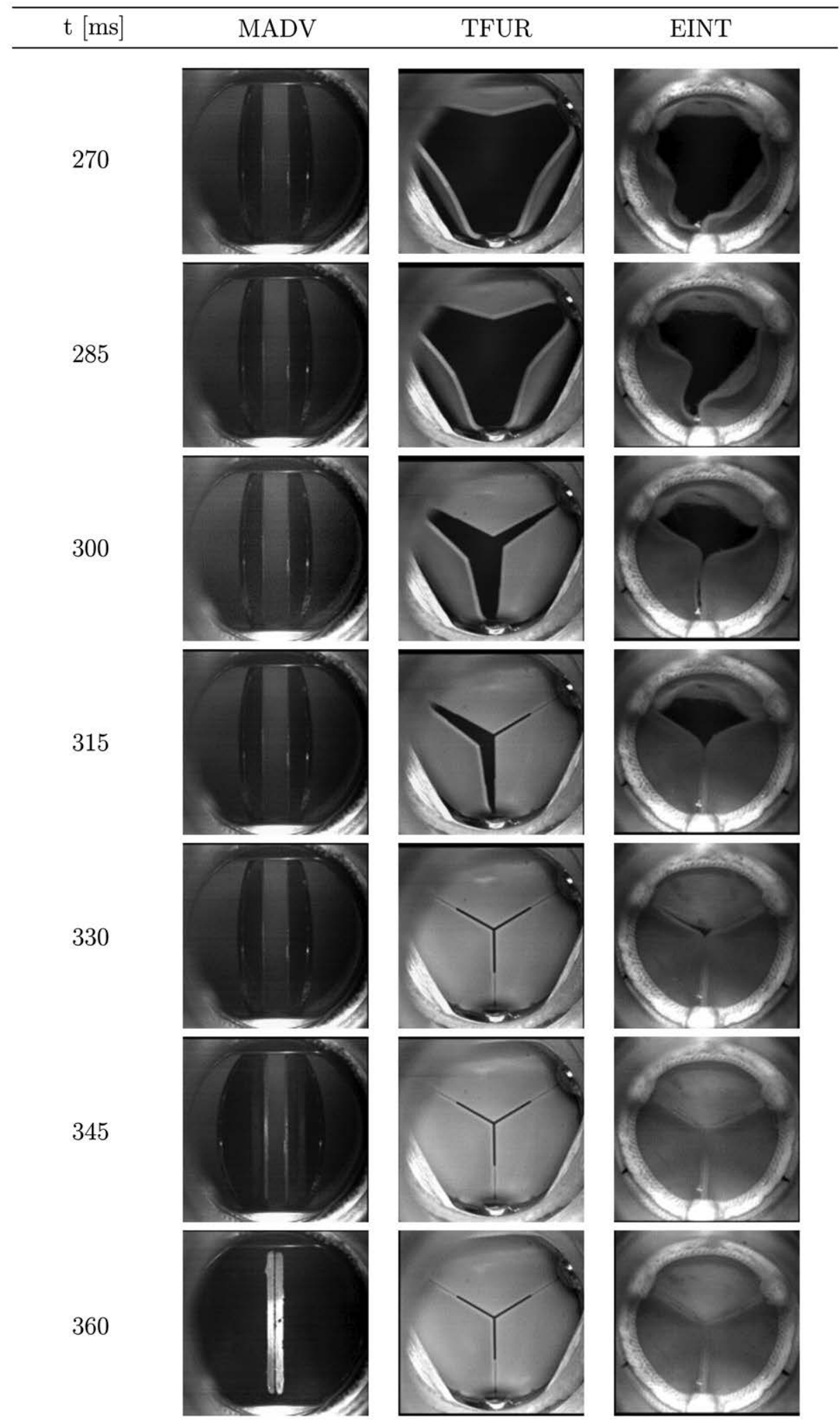

Figure 6. Qualitative comparison of the closing phase. Edwards INTUITY (EINT) and Lapeyre-Triflo FURTIVA (TFUR) closed early and slowly. Medtronic ADVANTAGE (MADV) closed later and more abruptly. 


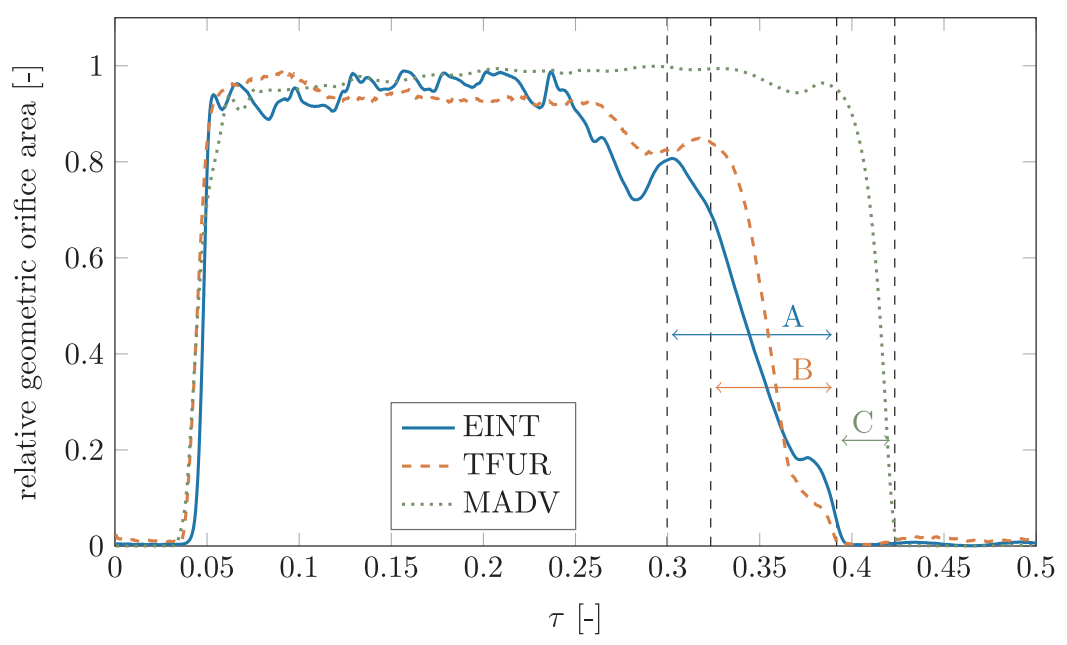

Figure 7. Relative geometric orifice area as function of nondimensionalized time with rapid valve closing time indicated. A: RVCT-EINT; B: RVCT:TFUR; C: RVCT-MADV. Medtronic ADVANTAGE (MADV) closed later and more abruptly than Edwards INTUITY (EINT) and Lapeyre-Triflo FURTIVA (TFUR). RVCT, rapid valve closing time.

\section{Individual Leaflet Kinematics: Mechanical Valves}

Figure 9 depicts a representative example of the leaflet opening angles and the leaflet tip velocities of the individual leaflets of TFUR and MADV (statistical results are given in Table 2, Supplemental Digital Content, http://links.Iww.com/ ASAIO/A184). The onset of opening was nearly identical at $\tau=0.036$. Full valve opening was achieved at $\tau=0.061$ for MADV and $\tau=0.058$ for TFUR, which translates to opening times of 20.0 and $18.5 \mathrm{~ms}$, respectively. The maximum opening velocity at the leaflet tips was $0.66 \mathrm{~m} / \mathrm{s}$ for MADV and $0.77 \mathrm{~m} / \mathrm{s}$ for TFUR for all leaflets. Leaflet 1 of TFUR closed by approximately 5 degrees at $\tau=0.1$, shortly after initial valve opening, whereas leaflets 2 and 3 remained in their fully open position. Rapid valve closure of TFUR started at $\tau=0.32$ when all three leaflets closed continuously with maximum tip velocities of approximately of $0.39 \mathrm{~m} / \mathrm{s}$. The closing phase of TFUR showed more variance with respect to the onset of leaflet motion of the individual leaflets than the opening phase. Leaflet 1 started closing considerably earlier than leaflets 2 and 3 . However, this did not affect the maximum leaflet tip velocities. The leaflet kinematics of MADV was very synchronous. After initial opening, the leaflets remained in the fully open position until the onset of valve closure at $\tau=0.39$. Both leaflets closed synchronously with maximum tip velocities of

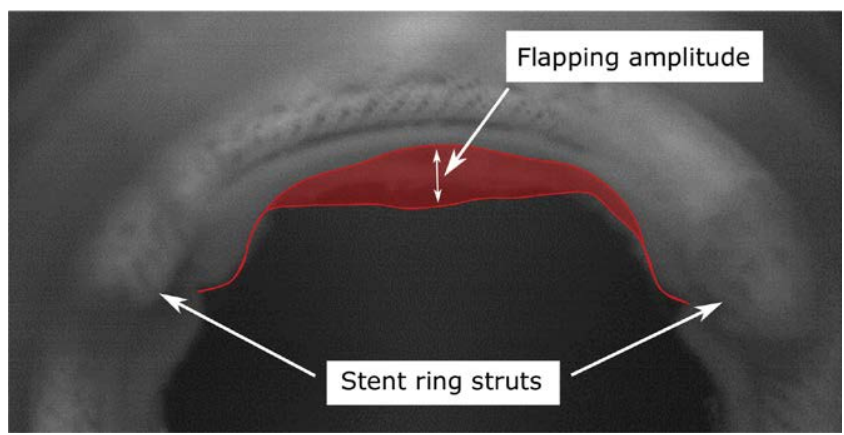

Figure 8. Flapping amplitude of Edwards INTUITY (EINT) leaflet fluttering motion. approximately $0.83 \mathrm{~m} / \mathrm{s}$. No leaflet rebound was observed for any of the tested valves.

\section{Individual Leaflet Kinematics: Bioprosthetic Valve}

Figure 10 depicts the leaflet kinematics of EINT at several instances during the cardiac cycle as indicated in the top panel. EINT opened synchronously starting at $\tau=0.035$ and ending at $\tau=0.053$, resulting in an opening time of $15 \mathrm{~ms}$. The maximum leaflet velocity was achieved during mid-opening at $\tau=0.048$. Here, the leaflets could reach a maximum local velocity of $2.03 \mathrm{~m} / \mathrm{s}$ (Figure 10C). This was also where the leaflets experienced the strongest bending during opening. The leaflet motion decelerated toward the end of valve opening (Figure 10, D-E). The rapid closure was more asynchronous starting at $\tau=0.298$ and ending at $\tau=0.397$. The maximum local leaflet velocity during closure was approximately 0.37 $\mathrm{m} / \mathrm{s}$ (statistical results for maximum opening and closing velocity are given in Table 2, Supplemental Digital Content, http:// links.Iww.com/ASAIO/A184). Leaflets 2 and 3 started closing first together with an inward bending of the leaflets (Figure 10, G-J). There was a short moment of stagnating leaflet motion (Figure 10K) before leaflet 1 closed fully (panel I). The local leaflet velocities were usually highest in the center of each leaflet, whereas the leaflet velocities were lowest close to the stent ring struts. Magnitudes and instances of the maximum local leaflet velocity for each leaflet, together with the average leaflet velocity at that instance, are indicated in Figure $\mathbf{9 .}$

\section{Reproducibility of the Results}

The reproducibility of the presented results was assessed by calculating the median deviation of the maximum leaflet velocities during opening and closure from their median value for each valve. This metric can be understood as the spread of the individual measurements about their median where lower values correspond to better reproducibility. The measurements for TFUR had a median deviation of $3.0 \%$. MADV and EINT had a median deviation of $6.3 \%$ and $7.4 \%$, respectively. The obtained values indicate good reproducibility of the given 

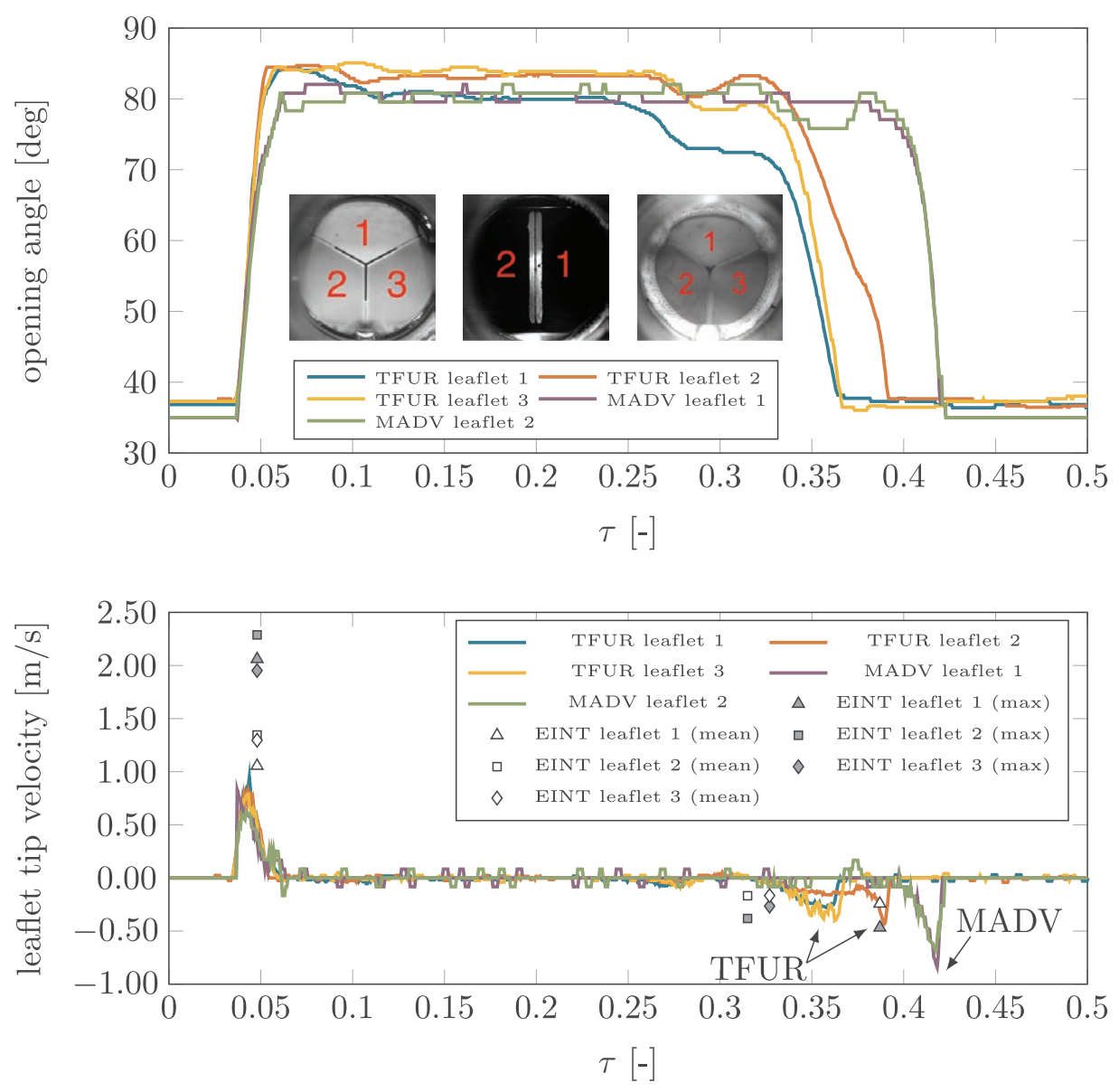

Figure 9. Opening angle and leaflet kinematics of Lapeyre-Triflo FURTIVA (TFUR) and Medtronic ADVANTAGE (MADV). Maximum in-plane leaflet velocity of Edwards INTUITY (EINT). MADV closed later than EINT and TFUR with increased leaflet tip velocity.

maximum leaflet velocities, with variations between individual measurements well below $10 \%$.

\section{Discussion}

The analysis of rGOA and the individual leaflet kinematics indicates that mechanical AVs reveal their most significant kinematic differences in the closing phase, which is most likely because of the generation of different flow fields in the aortic root, leading to different pressure fields acting on the leaflets. The opening phase, on the other hand, proved to be very similar for MADV and TFUR. EINT opened faster than the mechanical valves (as indicated by the opening times and the leaflet velocities), which may result in decreased flow resistance (and thereby a reduced pressure gradient) during early systole.

The slightly asynchronous motion of the TFUR may result from the orientation of the valve with respect to the direction of gravity, which acts on leaflet 1 in the closing direction, but in the opening direction for leaflets 2 and 3. Therefore, leaflet 1 closed slightly and had a lower opening angle throughout systole. This effect was confirmed by changing the orientation of TFUR, such that leaflets 2 or 3 were in the top position with respect to gravity, resulting in a slight closing of leaflet 2 (or 3, respectively) just after opening. Similar behavior was previously reported. ${ }^{15}$ The more synchronous behavior of the MADV can be partly attributed to the orientation of the valve where both leaflets were equally influenced by gravity. The asynchronous leaflet closure of EINT is frequently observed in experimental studies and may originate from local differences in the clamping of the flexible stent frame or slight mechanical differences between the leaflets, as was hypothesized for the asymmetrical leaflet fluttering.

Early and smooth closure of AV prostheses reduces the closing volume and lowers the risk of a high-velocity regurgitant jet, which can create high levels of shear stress in the blood. ${ }^{27}$ EINT and TFUR showed early and smooth closure, and therefore, a lower closing volume and hemodynamic benefits compared with MADV are expected. ${ }^{1,28}$

Rapid valve closure is the most critical phase with respect to mechanical leaflet stress. ${ }^{29,30}$ A prolonged RVCT gives more time for valve closure with reduced leaflet velocity and reduced RVCS. EINT showed the longest RVCT, followed by TFUR and MADV (see Table 1, Supplemental Digital Content, http://links. Iww.com/ASAIO/A183). Consequently, the leaflet tip velocities during rapid valve closure were considerably higher for MADV than those for TFUR and EINT. Therefore, higher mechanical loads on the leaflets of MADV and an increased risk of cavitation are expected compared with TFUR and EINT. This is well reflected in the nondimensional RVCS, penalizing abruptly closing valves.

The values for RVCT are generally in good agreement with previous experimental work. ${ }^{12,14,15,31}$ However, the values 

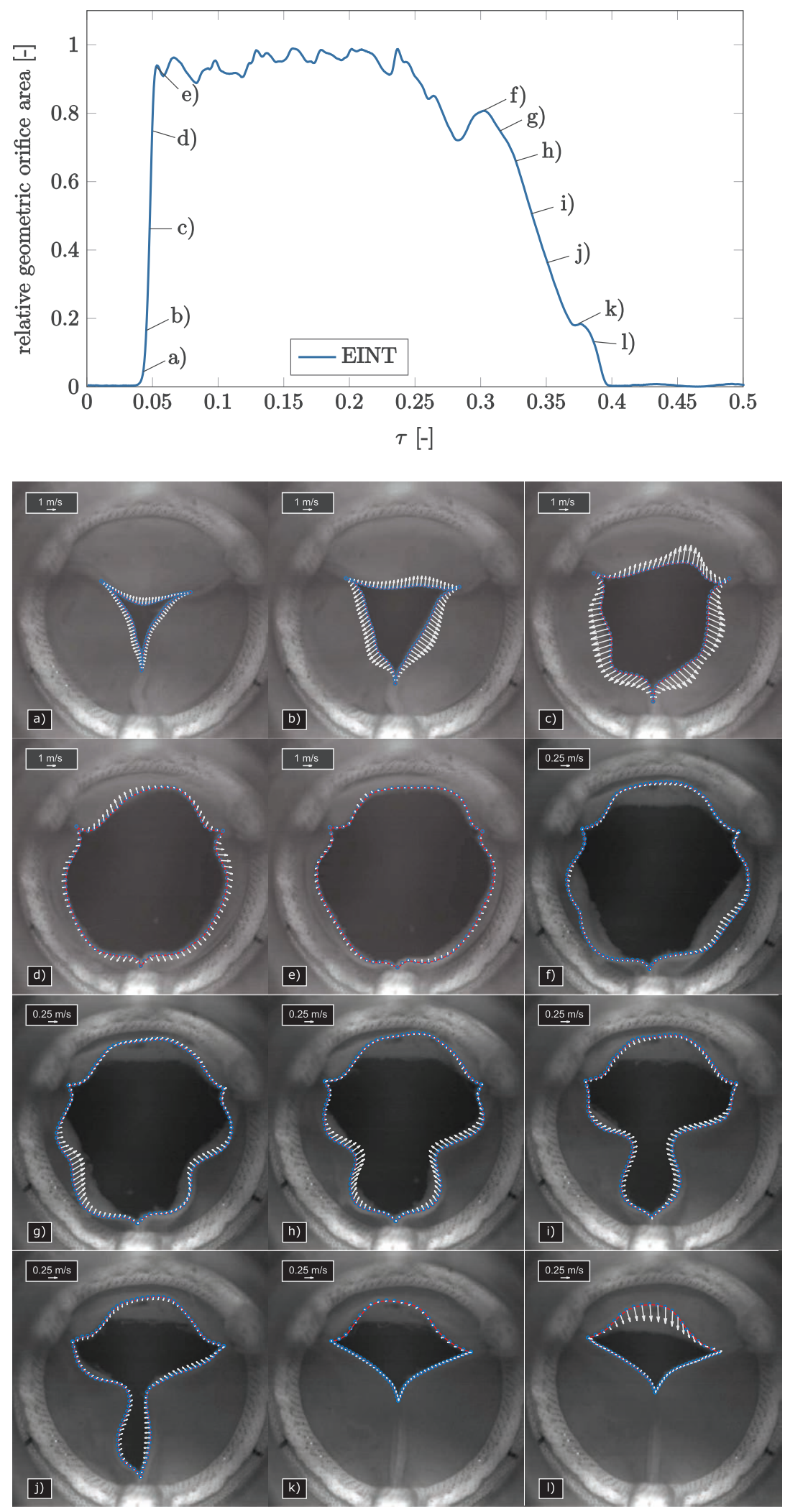

Figure 10. Individual leaflet kinematics of Edwards INTUITY (EINT) during opening (A-E) and closure (F-I). Maximum leaflet velocity during opening exceeded closing velocity by a factor of five. 
given in the literature can vary strongly among different studies because of differences in the experimental setups, making it difficult to quantitatively compare results.

The leaflet fluttering of bioprosthetic valves may contribute to the limited durability of these valves because it introduces additional mechanical load cycles on the leaflets during systole because of the repeated bending of the leaflet material in addition to the forces experienced during opening and closure.

\section{Limitations}

The main limitation of the presented technique lies in the ability of the in vitro setup to replicate the in vivo situation. While providing a controlled measurement environment, factors such as intercycle variability and the full biomechanical interaction of the valve with the surrounding tissue are not included. However, as the main focus of this study lies in the comparison of various AV designs under the same measurement conditions, this will not compromise the presented results. Additionally, it cannot be excluded that some effects seen in this study may be unique to the flow loop used and may not appear to the same degree in the human body. The analysis of the individual leaflet kinematics of EINT was limited to the dominant in-plane velocity component. This is to be taken as a lower bound for the true leaflet velocity. Resolving the full 3D motion of the leaflets was not possible with the current technique because of the complex leaflet motion of bioprosthetic valves. Resolving the 3D leaflet motion would require 3D imaging and subsequent surface reconstruction. ${ }^{32,33}$ This was beyond the scope of this work and may be topic of future investigations.

\section{Conclusion}

The use of an identical experimental setup allowed to directly compare three different aortic prostheses. A strong similarity in the kinematics between EINT and TFUR was found qualitatively and quantitatively. The kinematic differences between the two mechanical valves (TFUR and MADV) were significant. Although showing only small deviations during valve opening, the onset of valve closure was considerably earlier for all leaflets of TFUR compared with MADV. RVCS was used as a metric for characterizing rapid valve closure. The maximum leaflet tip velocities of MADV $(0.83 \mathrm{~m} / \mathrm{s})$ were significantly higher than those of TFUR $(0.39 \mathrm{~m} / \mathrm{s})$ and those of EINT $(0.37 \mathrm{~m} / \mathrm{s})$ during closure. During opening, the opposite situation was found with EINT $(2.03 \mathrm{~m} / \mathrm{s})$, showing significantly higher leaflet velocities than MADV $(0.66 \mathrm{~m} / \mathrm{s})$ and TFUR $(0.77 \mathrm{~m} / \mathrm{s})$. Early and smooth valve closure may indicate hemodynamic benefits of TFUR over MADV, whereas the rapid opening of EINT suggests hemodynamic benefits over the mechanical valves. The presented technique proved to be a valuable addition to the common measures and is able to yield more detailed information about the kinematics of AVs. In summary, bioprosthetic valves, such as EINT, appear to still exhibit the best kinematics among today's AV prostheses, but newly emerging mechanical heart valve designs seem to be closing the gap.

\section{Acknowledgments}

The authors thank Novostia SA (Neuchâtel, Switzerland) for providing us with the Lapeyre-Triflo FURTIVA valve used in this project and the Department of Cardiovascular Surgery of the University Hospital Bern for providing us with the Medtronic ADVANTAGE and the Edwards INTUITY valve. Furthermore, the authors thank Silje E. Jahren for the design of the flow loop and her assistance in this project. This work was funded through internal funds from ETH Zürich and the University of Bern.

\section{References}

1. Dasi LP, Simon HA, Sucosky P, Yoganathan AP: Fluid mechanics of artificial heart valves. Clin Exp Pharmacol Physiol 36: 225-237, 2009.

2. Johansen P, Andersen TS, Hasenkam JM, Nygaard H, Paulsen PK: Mechanical heart valve cavitation in patients with bileaflet valves. Conf Proc IEEE Eng Med Biol Soc 2014: 5655-5658, 2014.

3. Lee H, Akagawa E, Tatsumi E, Taenaka Y: Characteristics of cavitation intensity in a mechanical heart valve using a pulsatile device: synchronized analysis between visual images and pressure signals. J Artif Organs 11: 60-66, 2008.

4. Linde T, Hamilton KF, Navalon EC, Schmitz-Rode T, Steinseifer $\mathrm{U}$ : Aortic root compliance influences hemolysis in mechanical heart valve prostheses: an in-vitro study. Int J Artif Organs 35: 495-502, 2012.

5. Lo CW, Chen SF, Li CP, Lu PC: Cavitation phenomena in mechanical heart valves: studied by using a physical impinging rod system. Ann Biomed Eng 38: 3162-3172, 2010.

6. Thubrikar MJ, Skinner JR, Eppink RT, Nolan SP: Stress analysis of porcine bioprosthetic heart valves in vivo. I Biomed Mater Res 16: 811-826, 1982.

7. Thubrikar MJ, Nolan SP, Aouad J, Deck JD: Stress sharing between the sinus and leaflets of canine aortic valve. Ann Thorac Surg 42: 434-440, 1986.

8. Leyh RG, Schmidtke C, Sievers HH, Yacoub MH: Opening and closing characteristics of the aortic valve after different types of valve-preserving surgery. Circulation 100: 2153-2160, 1999.

9. Weston MW, LaBorde DV, Yoganathan AP: Estimation of the shear stress on the surface of an aortic valve leaflet. Ann Biomed Eng 27: 572-579, 1999.

10. Vennemann BM, Rösgen T, Carrel TP, Obrist D: Time-resolved micro PIV in the pivoting area of the triflo mechanical heart valve. Cardiovasc Eng Technol 7: 210-222, 2016.

11. Kasprzak JD, Nosir YF, Dall'Agata A, et al: Quantification of the aortic valve area in three-dimensional echocardiographic data sets: analysis of orifice overestimation resulting from suboptimal cut-plane selection. Am Heart J 135(6 pt 1): 995-1003, 1998.

12. Kuehnel RU, Pohl A, Puchner R, et al: Opening and closure characteristics of different types of stented biological valves. Thorac Cardiovasc Surg 54: 85-90, 2006.

13. Tasca G, Fiore GB, Mangini A, et al: Opening-closing pattern of four pericardial prostheses: results from an in vitro study of leaflet kinematics. J Artif Organs 19: 350-356, 2016.

14. Wendt D, Stühle S, Marx P, et al: The investigation of systolic and diastolic leaflet kinematics of bioprostheses with a new in-vitro test method. Minim Invasive Ther Allied Technol 24: 274-281, 2015.

15. Brücker, C, Steinseifer, U, Schröder, W, Reul, H: Unsteady flow through a new mechanical heart valve prosthesis analysed by digital particle image velocimetry. Meas. Sci. Technol. 13, 1043-1049, 2002.

16. Gallegos RP, Rivard AL, Suwan PT, et al: In-vivo experience with the Triflo trileaflet mechanical heart valve. J Heart Valve Dis 15: 791-799, 2006

17. Kaufmann TA, Linde T, Cuenca-Navalon E, et al: Transient, threedimensional flow field simulation through a mechanical, trileaflet heart valve prosthesis. ASAIO / 57: 278-282, 2011.

18. Li CP, Lu PC: Numerical comparison of the closing dynamics of a new trileaflet and a bileaflet mechanical aortic heart valve. J Artif Organs 15: 364-374, 2012.

19. Sato M, Harasaki H, Wika KE, Soloviev MV, Lee AS: Blood compatibility of a newly developed trileaflet mechanical heart valve. ASAIO J 49: 117-122, 2003. 
20. Steinseifer, U, Schmitz, E, Linde, T, Kaufmann, T, Schmitz-Rode, T: The Triflo trileaflet mechanical heart valve: design and in vitro performance. Int. J. Artif. Organs, 32: 400-400, 2009.

21. Jahren SE, Winkler BM, Heinisch PP, Wirz J, Carrel T, Obrist D: Aortic root stiffness affects the kinematics of bioprosthetic aortic valves. Interact Cardiovasc Thorac Surg 24: 173-180, 2017.

22. Robicsek F, Thubrikar MJ: Role of sinus wall compliance in aortic leaflet function. Am / Cardiol 84: 944-946, A7, 1999.

23. Sripathi VC, Kumar RK, Balakrishnan KR: Further insights into normal aortic valve function: role of a compliant aortic root on leaflet opening and valve orifice area. Ann Thorac Surg 77: 844-851, 2004

24. Swanson M, Clark RE: Dimensions and geometric relationships of the human aortic valve as a function of pressure. Circ Res 35: 871-882, 1974

25. Barnea, DI, Silverman, HF: Class of algorithms for fast digital image registration. IEEE T. Comput C21: 179-186, 1972.

26. Scotten LN, Siegel R: Importance of shear in prosthetic valve closure dynamics. J Heart Valve Dis 20: 664-672, 2011.

27. Scotten LN, Walker DK: New laboratory technique measures projected dynamic area of prosthetic heart valves. J Heart Valve Dis 13: 120-132; discussion 132, 2004.
28. Lu PC, Liu JS, Huang RH, Lo CW, Lai HC, Hwang NH: The closing behavior of mechanical aortic heart valve prostheses. ASAIO J 50: 294-300, 2004.

29. Fries R, Graeter T, Aicher D, et al: In vitro comparison of aortic valve movement after valve-preserving aortic replacement J Thorac Cardiovasc Surg 132: 32-37, 2006.

30. Katayama S, Umetani N, Sugiura S, Hisada T: The sinus of Valsalva relieves abnormal stress on aortic valve leaflets by facilitating smooth closure. I Thorac Cardiovasc Surg 136: 1528-1535, 1535.e1, 2008.

31. Romagnoni C, Mangini A, Vismara R, et al: Aortic interleaflet triangles reshaping: hydrodynamic, kinematic and morphological effects in in-vitro analysis. I Heart Valve Dis 23: 424-431, 2014

32. Gunning PS, Saikrishnan N, Yoganathan AP, McNamara LM: Total ellipse of the heart valve: the impact of eccentric stent distortion on the regional dynamic deformation of pericardial tissue leaflets of a transcatheter aortic valve replacement. J $R$ Soc Interface 12: 20150737, 2015

33. Iyengar AKS, Sugimoto $H$, Smith DB, Sacks MS: Dynamic in vitro quantification of bioprosthetic heart valve leaflet motion using structured light projection. Ann Biomed Eng 29: 963-973, 2001. 